\title{
Sind die „Sommerzellen“ in der Nebenniere des Frosches acidophil?
}

Von

\author{
Dr. Walter Carl, \\ Privatdozenten an der Universitit Königsberg i. Pr.
}

Hierza 1. Textfigur.

In den Nebennieren des Frosehes liegen nebeneinander die verschiedenen Arten der Zellen, welche wir bei den höheren Tieren, vor allem bei den Säugern, von einander getrennt und topographisch auf die Rinden- und Markschicht verteilt vorfinden. Von diesen. Zellelementen haben die von H. Stilling (1) als eine Gattung sui generis einst beschriebenen "Sommerzellen" eine besondere Beachtung gefunden. Wie der Name besagen soll, handelt es sich um Zellen, deren Vorkommen an eine bestimmte Jahreszeit gebunden schien; diese Feststellung glaubte ihr Entdecker, der den Zellen auch den Namen gegeben hat, mit Bestimmtheit machen zu können. Von späteren Untersuchern wurde das Vorkommen dieser Zellen bestătigt, aber sie fanden, dass das Vorkommen nicht an den

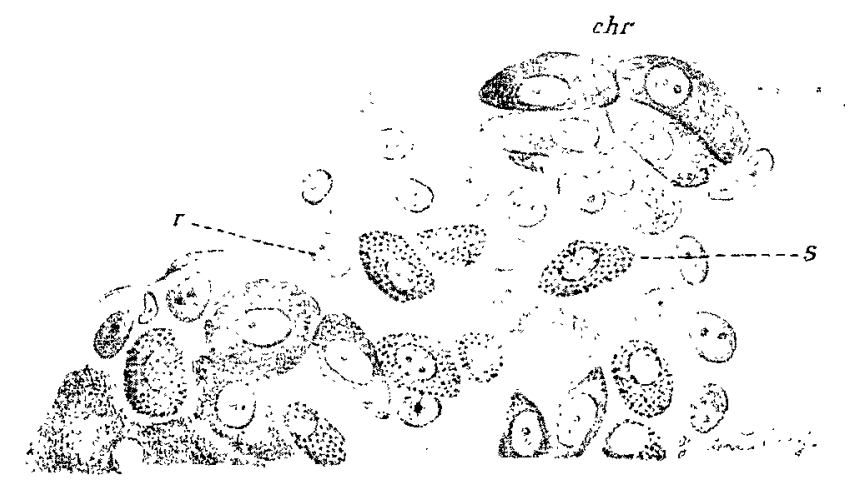

Nebennierenstränge eines Winterfrosches (Rana escul.). Fixierung nachWies sel, Färbung mit Wasserblau und Safranin.

$\mathrm{r}=$ Rindenzelle. $\mathrm{chr}=$ chromaffine Zelle. $\mathrm{s}=$ Zelle mit safraninophilen Granulis. Vergr. 500fach. 
Sommer gebunden war, sondern dass die Zellen auch in Wintertieren vorkamen. Patzelt und $\mathrm{K}$ ubik (2) haben zu allen Jahreszeiten bei der Gattung Rana esc. Sommerzellen nachweisen können, und zwar haben sie mit besonderer Sorgfalt darauf geachtet, dass die von ihnen untersuchten Tiere sich auch tatsächlich in dem Winterzustande befanden. Es ist angezweifelt worden, dass im Laboratorium gehaltene Tiere sich in demselben physiologischen Zustande befinden, wie die in der Freibeit. Die Untersucher haben deshalb im Winterschlaf befindliche Tiere direkt ausgegraben. Auch in solchen konnten sie die Stilling schen Zellen, wie sie angeben, in unverminderter Zahl und Färbbarkeit finden. Patzelt und K u bik schlagen vor, den Namen "Sommerzellen" aufzugeben und dafür die Bezeichnung ,acidophile" Zellen einzuführen, da die Granula der Zellen eine besondere Affinität zu sauren Farbstoffen haben, wie durch Anwendung von Eosinlösung, dem E h r li c hBi ond i schen Dreifarbengemisch, von Orange, Erythrosin, Säurefuchsin bewiesen werden konnte. Nur die acidophilen (eosinophilen) Leukozyten des Blutes sollten eine noch grössere Verwandtschaft zu sauren Farbstoffen besitzen.

Die Kenntnis dieser acidophilen Zellen glaube ich auf Grund meiner Untersuchungen ein wenig erweitern zu können.

Meine ersten Untersuchungen gehen zurück bis zum Winter 1910/11; gelegentlich meiner Studien über das chromaffine System (4), fielen mir auch die acidophilen Zellen auf. Ich behandelte die zu untersuchenden Nebennieren nach der von Wi es el (3) angegebenen Nethode. Ich konnte mein Material immer lebensfrisch fixieren und habe meistens, um eine gute Chromierung zu erzielen, die Fisierung im Brutschrank bei $30^{\circ}$ bis $37^{\circ}$ sich vollzieben lassen. Bei Nachfürbung der Präparate mit 1 Prozent Safraninlösung zur Darstellung der Kerne fiel mir auf, dass sich in den Nebennieren einzelne Zellen in ihrem Plasmateil rot färbten und dass diese Rotfärbung bei stärkerer Vergrösserung sich als eine exakte Granulafärbung erkennen liess. Es wurden von mir drei Winter hindurch immer eine Anzahl von Fröschen untersucht mit dem gleichen Resultat. Vergleiche meiner Präparate, die von männlichen und weiblichen Tieren vom Sommer und Winter stammten, mit den von Patzelt und $K u$ bik gegebenen Darstellungen der acidophilen Zellen lassen keinen Zweifel darüber, dass es sich um identische Zellen handelt. Die Anordnung in den Nebennierensträngen, die 
Zabl und Form der Zellen, sowie die Art der Granulierung zeigen weitgehende Übereinstimmungen; die Granula scheinen mir in der Darstellung mit Safranin noch distinkter als mit Eosin. Eine Beziehung dieser Gebilde zu dem Ernahrungszustand und zu der Geschlechtstatigkeit konnte ich nicht finden.

Safranin ist ein basophiler Farbstoff. Wenn sich die Granulationen der Sommerzellen so, wie ich es gefunden habe, mit einem basophilen Farbstoff färben lassen, kann man nicht gut an der Bezeichnung acidophil festhalten. Mit Ausnahme dieser Granula ist das Plasma irgend welcher anderer Zellen in meinen Präparaten durch Safranin nicht gefarbt, wohl zeigen aber die Zellkerne, besonders die Kerne der roten Blutkörperchen eine distinkte Tinktion mit Safranin.

\section{Literatur.}

1. Gaupp: Anatomie des Frosches. 2. Aufl.

2. Patzelt, V. a. Kubik, J.: Acidophile Zellen in der Nebenniere von Rana escul. Arch. f. mikr. Anat., Bd. 81, S. 82-91.

3. Schmorl, G. : Untersuchungsmethoden. 7. Aufl, 1914, S. 293.

4. Car 1: Das chromaffine System und seine Erschöpfung durch Muskelarbeit. Deutsch. med. Woch. 1911, Nr. 40. 